ANNALS OF

CLINICAL

NEUROPHYSIOLOGY

\title{
Paraneoplastic demyelination in the brain presenting as a clinically occult non-Hodgkin's lymphoma
}

\author{
Hyoshin Son ${ }^{1 *}$, Jongsuk Choi ${ }^{2 *}$, Sung Un Kim², and Kyung Seok Park ${ }^{2}$ \\ 1 Department of Neurology, Seoul National University Hospital, Seoul National University College of Medicine, \\ Seoul, Korea \\ ${ }^{2}$ Department of Neurology, Seoul National University Bundang Hospital, Seoul National University College of \\ Medicine, Seongnam, Korea
}

Received: March 27, 2019

Revised: July 1, 2019

Accepted: July 2, 2019

\section{Correspondence to}

Kyung Seok Park

Department of Neurology, Seoul National University Bundang Hospital, 82 Gumi-ro 173 Beon-gil, Bundang-Gu, Seongnam

13620, Korea

Tel: +82-31-787-7466

Fax: $+82-31-787-4059$

E-mail:kpark78@naver.com

*Both authors contributed equally to the work

\section{ORCID}

\section{Hyoshin Son}

https://orcid.org/0000-0001-6643-3712

Jongsuk Choi

https://orcid.org/0000-0003-0498-5401

Sung Un Kim

https://orcid.org/0000-0003-4909-5218

Kyung Seok Park

https://orcid.org/0000-0003-1553-5932

\section{CASE}

A previously healthy 40-year-old woman was admitted to the emergency department for

Non-Hodgkin's lymphoma (NHL) may initially present with atypical neurological manifestations, including paraneoplastic neurological syndromes. Herein, we report the case showing an initial manifestation of systemic NHL with paraneoplastic demyelination in the brain that initially mimicked the symptoms of stroke, seizure, and brain tumor. A high index of suspicion and timely diagnostic workup is required to prevent diagnostic delay and commence proper management of the condition. In this situation, a whole-body FDG PET/CT could be useful to screen for occult malignancy.

Key words: Paraneoplastic syndrome; Occult malignancy; Demyelinating diseases; Non-Hodgkin lymphoma; Diffuse large B-cell lymphoma

Non-Hodgkin's lymphoma (NHL) usually manifests as painless lymphadenopathy with occasional systemic symptoms such as fever, weight loss, and night sweats. ${ }^{1}$ However, initial atypical presentations in the form of paraneoplastic neurological syndromes (PNSs) may occur before the cancer is diagnosed. ${ }^{2,3}$ PNSs occur in less than $1 \%$ of patients with solid tumors, a frequency that is likely lower among patients with lymphomas., Early diagnosis and treatment of the underlying cancer are important for achieving a clinically successful outcome. Herein, we report the case of a woman who presented with paraneoplastic demyelination in the brain as an initial manifestation of systemic large B-cell NHL, which initially mimicked the symptoms of stroke, seizure, and brain tumor.

This is an Open Access article distributed under the terms of the Creative Commons Attribution Non-Commercial License (http:// creativecommons.org/licenses/by-nc/4.0) which permits unrestricted non-commercial use, distribution, and reproduction in any medium, provided the original work is properly cited. 
recurrent right hemiparesis, visual field loss, dysarthria, and throbbing headaches that had worsened over the course of 1 week. On admission, the patient had a regular pulse rate of 92 beats per minute and blood pressure of 129/83 mmHg. Neurological examination revealed right-side hemiparesis (Medical Research Council grade 4) and left-side binocular homonymous hemianopia. Diffusion-weighted magnetic resonance imaging (MRI) and electroencephalography (EEG) revealed no abnormalities. Laboratory test results were within normal ranges, except for a mild elevation of serum lactate dehydrogenase (282 IU/L). By day 3 of hospital stay, all the symptoms had resolved without treatment; however, right hemiparesis and dysarthria recurred on day 7. MRI performed on the same day revealed multifocal confluent subcortical lesions on T2-weighted and fluid-attenuated inversion recovery images without definite contrast enhancement (Fig. 1A). Diffusion-weighted MRI revealed increased apparent diffusion coefficient in the involved brain lesions. Cerebral angiography revealed no steno-occlusive lesions or vascular malformations.

The differential diagnoses included low-grade brain tumor and demyelination. Cerebrospinal fluid (CSF) analysis revealed a protein concentration of $166.7 \mathrm{mg} / \mathrm{dL}$ (normal range $<45 \mathrm{mg} / \mathrm{dL}$ ) without cellularity and a normal CSF/ serum glucose ratio. Results of the CSF cultures and viral polymerase chain reaction studies were negative. Immunologic profiling for various antibodies, including neuromyelitis optica immunoglobulin $\mathrm{G}$ antibody, yielded negative

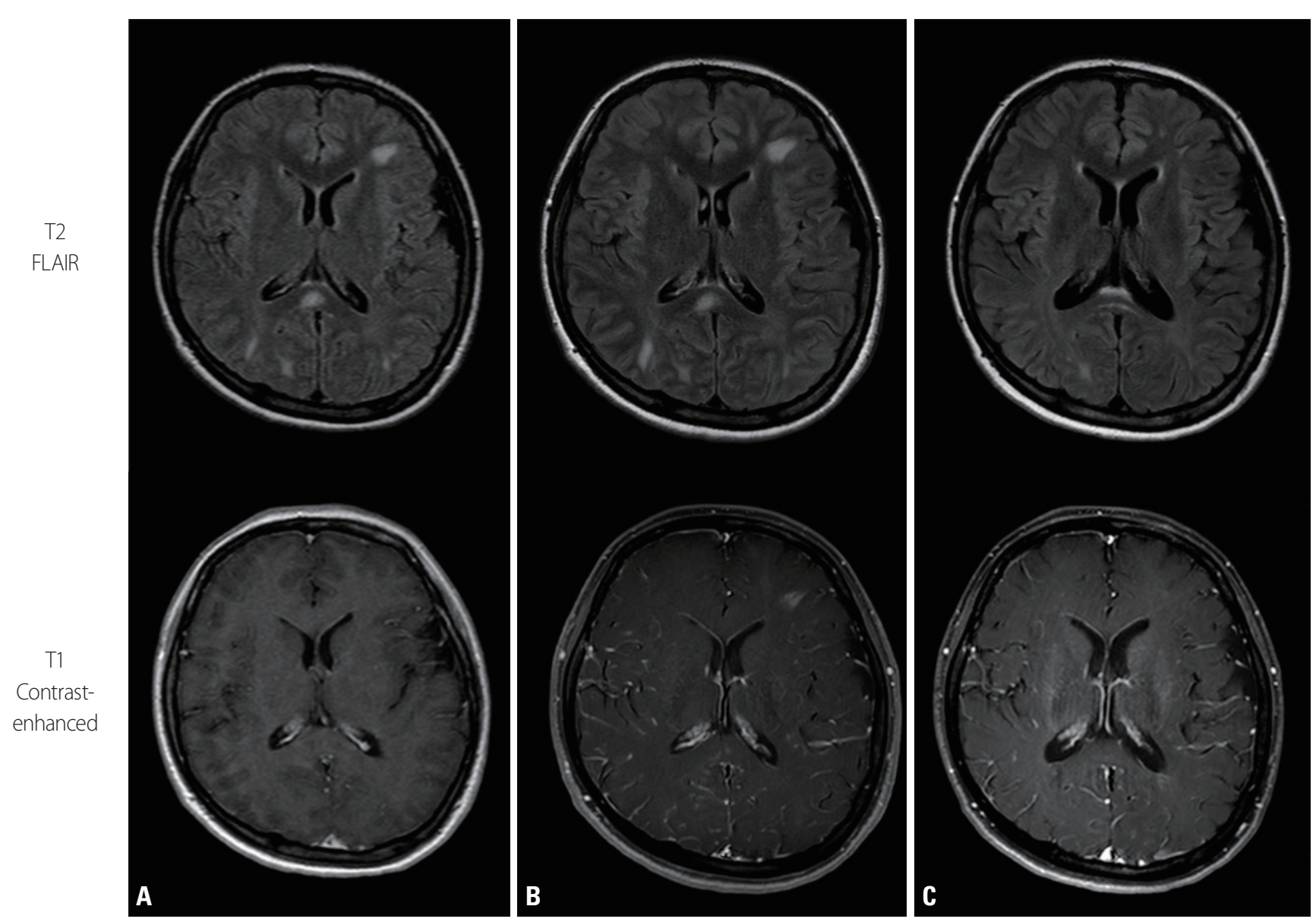

Fig. 1. Serial follow-up of magnetic resonance imaging (MRI) examinations was performed at (A) 2 weeks and (B) 1 month after symptom onset, as well as (C) 1 year following the initial cycle of chemotherapy. Bilateral involvement of the periventricular, subcortical, frontal, and parietooccipital white matter and the splenium of the corpus callosum on T2-weighted fluid-attenuated inversion recovery images are seen. While the initial MRI lacked contrast enhancement, it appeared on the follow-up MRI performed at 1 month after symptom onset. After chemotherapy, the extent of the lesion markedly decreased and the absence of contrast enhancement was observed. 
results. The paraneoplastic antibodies panel (anti-Hu, anti-Ri, anti-Yo, anti-amphiphysin, anti-CV2, anti-PNMA2, anti-Recoverin, anti-SOX1, and anti-Titin) was negative. Based on these findings, we presumptively rendered a diagnosis of central nervous system (CNS) demyelinating disease. The patient was treated intravenously with methylprednisolone at 1,000 mg/day for 5 days. She showed clinical improvement and was discharged.

The patient experienced an hour-long episode of transient amnesia without loss of consciousness in the first month after discharge. Follow-up EEG revealed left frontal dominance with diffuse slowing, which was indicative of regional cerebral dysfunction. When compared with the previous $M R I$ results, the follow-up MRI revealed a definite growth of the lesion with prominent contrast enhancement (Fig. 1B).
We considered possible malignancy, but the patient elected not to undergo stereotaxic biopsy. A follow-up CSF analysis revealed an increased protein concentration of $205.4 \mathrm{mg} / \mathrm{dL}$ as compared to the value in the previous study. Malignant cells were not found on the repeat cytologic evaluation of CSF. To evaluate the possibility of a secondary CNS tumor and existence of primary systemic cancer, we performed a chest-abdomen-pelvis computed tomography (CT) scan. $\mathrm{CT}$ examination revealed splenomegaly and an osteolytic lesion with contrast enhancement on $\mathrm{T} 7$ and $\mathrm{T} 12$ vertebrae (Fig. 2A). Whole-body including brain ${ }^{18}$ F-fluorodeoxyglucose (FDG) positron emission tomography (PET)/CT revealed multiple hypermetabolic lesions in the thoracic spine, bone marrow, and spleen (Fig. 2B). However, there was no significant alteration of brain metabolism. A pathologic study via
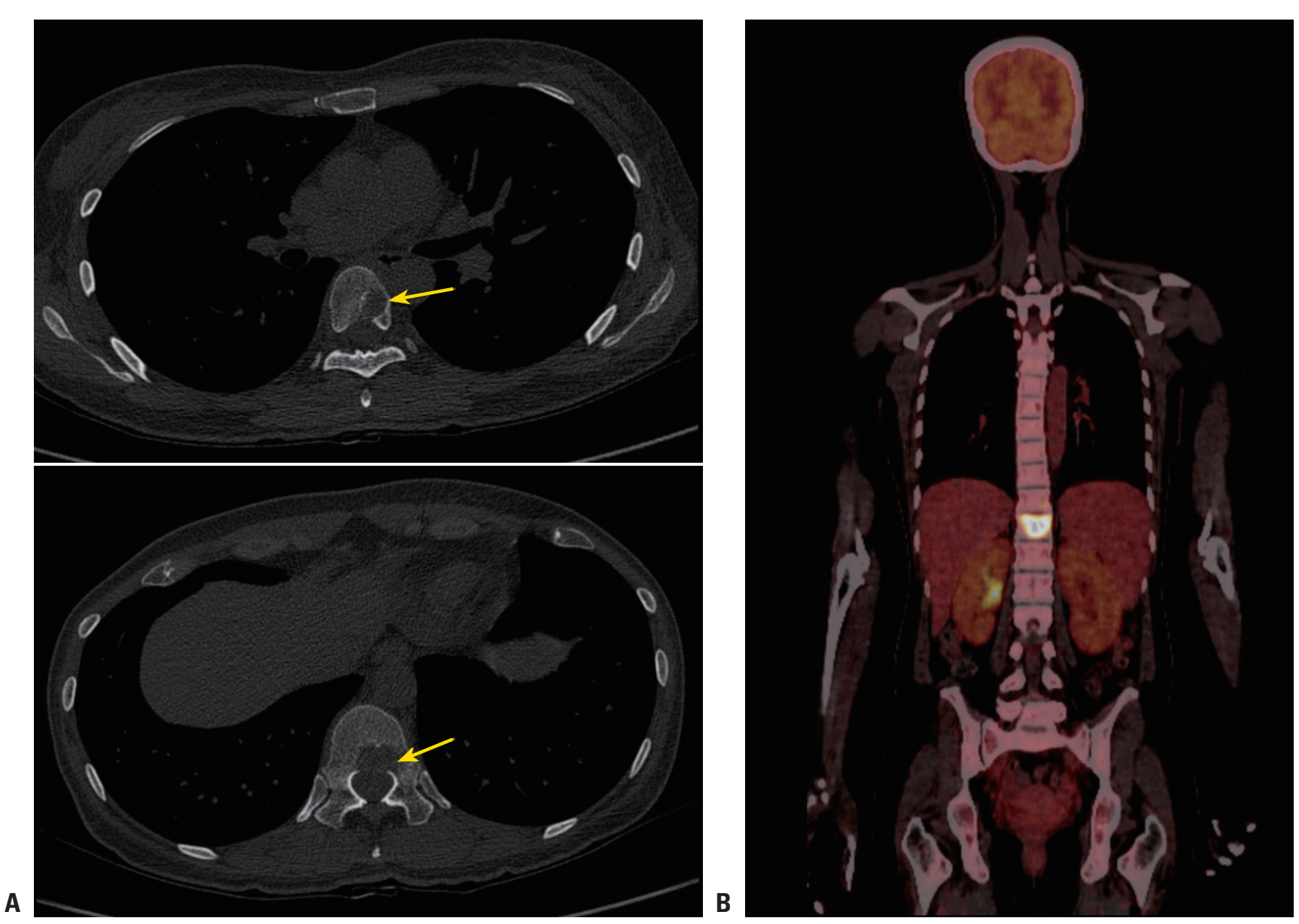

Fig. 2. Computed tomography (CT) revealed (A) an osteolytic lesion on T7 and T12 vertebral bodies (yellow arrows). Whole-body (including the brain) ${ }^{18} \mathrm{~F}$-fluorodeoxyglucose positron emission tomography/CT revealed (B) a hypermetabolic lesion in the thoracic spine, bone marrow, and spleen without significant alteration of brain metabolism. 
CT-guided bone biopsy of the thoracic vertebra revealed a malignant lymphoma with B-cell lineage. We rendered a diagnosis of $\mathrm{NHL}$ with paraneoplastic demyelination in the brain and began six cycles of rituximab, cyclophosphamide, doxorubicin, vincristine, and prednisolone chemotherapy as well as three cycles of intrathecal methotrexate/hydrocortisone for CNS prophylaxis. A follow-up brain MRI 1 year after the initial cycle of chemotherapy revealed that the extent of the lesion had markedly decreased (Fig. 1C), and there was no further recurrence of neurological symptoms.

\section{DISCUSSION}

PNSs are rare disorders that can affect any part of the nervous system of cancer patients. They are not caused by metastasis, direct invasion, or consequences of treatment. ${ }^{4}$ They usually result from an autoimmune response against the neuronal proteins expressed by cancer cells. ${ }^{4,5}$ The PNS Euronetwork established the diagnostic criteria for PNSs based on the presence or absence of cancer, "classical" syndromes, and "well-characterized" onconeural antibodies. Classical PNSs include encephalomyelitis, limbic encephalitis, subacute cerebellar degeneration, opsoclonus-myoclonus, subacute sensory neuronopathy, chronic gastrointestinal pseudo-obstruction, Lambert-Eaton myasthenic syndrome, and dermatomyositis. ${ }^{4,5}$ Our patient presented with neurological symptoms such as recurrent hemiparesis, visual field loss, dysarthria, and transient amnesia, which are non-classical manifestations of PNS. Although paraneoplastic brain demyelination is uncommon, several case reports have documented its occurrence. ${ }^{6-8}$ The MRI findings of these reports are almost identical to those of our patient, i.e., multifocal confluent subcortical lesions involving the corpus callosum without contrast enhancement. Follow-up brain MRIs of our patient after systemic chemotherapy with CNS prophylaxis showed markedly decreased lesions and clearly improved neurological symptoms. We diagnosed our patient with possible PNS in accordance with the diagnostic criteria of the PNS Euronetwork. ${ }^{5}$

Although our patient refused to undergo a brain biopsy that would have provided a definitive diagnosis, a wholebody (including the brain) FDG PET/CT allowed for the evaluation of metabolic activity that ruled out brain-related neoplastic conditions and identified an occult systemic malignancy. ${ }^{9}$ Repeated cytologic evaluation of the CSF also aided in diagnosis; the absence of malignant cells and the markedly increased protein levels suggested an active inflammatory or demyelinating process. ${ }^{10}$ While we were unable to find any paraneoplastic antibodies, PNSs may occur without onconeural antibodies; indeed, onconeural antibodies are absent in most cases of PNS associated with lymphoma. ${ }^{4}$ Hence, the use of onconeural antibodies to inform a definitive diagnosis of PNSs associated with lymphoma is limited.

The present report serves as a reminder that systemic NHL may be evinced by varied neurological manifestations and demyelinating brain lesion presenting as PNSs. In cases of recurrent and unexplained acute neurologic symptoms and unspecified brain lesions involving multifocal white matter, systemic NHL with paraneoplastic demyelination in the brain should be considered as a possible etiology to prevent diagnostic delay and commence proper management. In this situation, whole-body FDG PET/CT could be a useful screening tool for occult malignant disease.

\section{Conflicts of Interest}

The authors have no financial conflicts of interest.

\section{REFERENCES}

1. Armitage JO, Gascoyne RD, Lunning MA, Cavalli F. Non-Hodgkin lymphoma. Lancet 2017;390:298-310.

2. Rosenfeld MR, Dalmau J. Update on paraneoplastic neurologic disorders. Oncologist 2010;15:603-617.

3. Giometto B, Grisold W, Vitaliani R, Graus F, Honnorat J, Bertolini G, et al. Paraneoplastic neurologic syndrome in the PNS Euronetwork database: a European study from 20 centers. Arch Neurol 2010;67:330-335.

4. Graus F, Ariño H, Dalmau J. Paraneoplastic neurological syndromes in Hodgkin and non-Hodgkin lymphomas. Blood 2014;123:3230-3238.

5. Graus F, Delattre JY, Antoine JC, Dalmau J, Giometto B, Grisold W, et al. Recommended diagnostic criteria for paraneoplastic neurological syndromes. J Neurol Neurosurg Psychiatry 2004;75:113540.

6. Glantz MJ, Biran H, Myers ME, Gockerman JP, Friedberg MH. The 
radiographic diagnosis and treatment of paraneoplastic central nervous system disease. Cancer 1994;73:168-175.

7. Wong K, Poon P, Berry K, Coppin C, Kostashuk E. Paraneoplastic demyelinating disorder in the brain of a patient with seminoma. J Comput Assist Tomogr 1998;22:136-138.

8. Gonzales N, Jarboe E, Kleinschmidt-DeMasters BK, Bosque P. Acute multifocal CNS demyelination as first presentation of sys- temic malignancy. Neurology 2005;65:166.

9. Matsuhisa A, Toriihara A, Kubota K, Makino T, Mizusawa H, Shibuya H. Utility of F-18 FDG PET/CT in screening for paraneoplastic neurological syndromes. Clin Nucl Med 2012;37:39-43.

10. Pavlidis N. The diagnostic and therapeutic management of leptomeningeal carcinomatosis. Ann Oncol 2004;15 Suppl 4:iv285iv291. 